\title{
Omni-wedge technique for increased dose homogeneity in head and neck radiotherapy
}

\author{
F.L.R. Vinagre*, P.C.P.S. Simões, P.J.B.M. Rachinhas
}

Serviço de Radioterapia, Hospitais da Universidade de Coimbra, Praceta Prof. Mota Pinto, 3000-075 Coimbra, Portugal

Received 9 May 2008; received in revised form 26 January 2009; accepted 11 February 2009

\section{KEYWORDS}

Radiotherapy;

Treatment planning;

Head and neck;

Omni-wedge

\begin{abstract}
Dose homogeneity in head and neck tumour irradiation is a challenging task for conventional radiotherapy due to large anatomic contour irregularities and tissue heterogeneities in irradiation volumes. We present a simple technique based on the omni-wedge concept which uses superimposed fields, orthogonally wedged, to improve target-volumes homogeneity in head and neck tumour irradiation. The routine implementation of this technique is straightforward and easy to achieve with standard radiotherapy equipment and treatment planning software. Our five-year experience in the clinical implementation of this technique is presented.
\end{abstract}

(c) 2009 Associazione Italiana di Fisica Medica. Published by Elsevier Ltd. All rights reserved.

\section{Introduction}

Irradiation of head and neck tumours is one of the most demanding tasks for conventional radiotherapy. Treatment volumes not only involve the tumour primary site but also lymphatic regions that extend target volumes into regions that include large anatomic contour irregularities (mouth, shoulders) and significant tissue heterogeneities (air cavities, bone, soft tissue). Moreover, the relative shape and position of target volumes and organs at risk, particularly the spinal cord position, further increases treatment planning complexity.

In recent years, work has been done in three-dimensional conformal radiotherapy (3D-CRT), intensity-

\footnotetext{
* Corresponding author. Tel.: +351 239403960; fax: +351 239838868.
}

E-mail address: filipa@huc.min-saude.pt (F.L.R. Vinagre). modulated radiation therapy (IMRT) and proton radiotherapy to minimize critical organ doses while preserving target coverage. It has been shown that as far as the target coverage is concerned, there are small differences between proton and IMRT techniques when compared to conformal irradiation techniques [1-3]. On the other hand, the spinal cord (dose-limiting organ) and the parotid salivary glands (quality of life limiting organs), can strongly benefit from proton or IMRT techniques [1-3].

Customized physical compensator techniques have been used for decades to perform simple forms of intensity modulation in order to compensate anatomic contour irregularities and tissue inhomogeneity [4,5]. In the last years, customized physical compensators are also being used as an alternative approach to deliver IMRT treatments with the advantage of cost and simplicity [6].

It seems that IMRT techniques will become available in a large number of centres within a reasonable time frame. In the meantime, however, most of these techniques

1120-1797/\$ - see front matter @ 2009 Associazione Italiana di Fisica Medica. Published by Elsevier Ltd. All rights reserved. doi:10.1016/j.ejmp.2009.02.002 
remain limited to a small number of large centres, while standard techniques are still used in many more radiotherapy departments such as our own.

Traditionally, a three-field technique is used in head and neck radiotherapy, consisting of two bilateral parallelopposed fields to treat the primary site as well as the upper and mid-cervical nodes above the shoulders, and a third anterior field elected to treat the lower neck and supraclavicular lymph nodes, abutted to the lateral fields. Whenever possible, the junction longitudinal position is chosen outside the primary disease site to prevent tumour under-dosage but is conditioned by the patient shoulders relative position to the primary tumour site $[7,8]$. In most radiotherapy departments these fields are outlined during a simulation procedure on radiographic or fluoroscopic images and drawn on immobilization masks for easier patient set-up. Anatomic contour irregularities and tissue inhomogeneity can lead to significant dose inhomogeneity in the target volumes. Although the use of 3D-customized tissue compensators has proven to improve dose homogeneity $[4,5]$, their manufacturing is time-consuming and their use is not widespread.

We present a simple technique to improve targetvolumes homogeneity without the use of 3D-custom tissue compensators. To optimize the dose distribution, field wedging is possible but collimator rotation is limited to multiples of $90^{\circ}$ because a flat junction region for the abutted fields is a main concern. Our technique is based on the omni-wedge concept which splits each lateral field into two orthogonally wedged segments and one open segment.

The omni-wedge concept, developed by Milliken et al. $[9,10]$, consists of creating a wedged field with arbitrary angle and orientation relative to the collimator by combining two orthogonal wedged fields segments plus an open field segment at fixed collimator and gantry positions. This concept originated from the need to choose effective wedge angles and orientations with the constraints of prohibited collimator rotation in order to have a specific multi-leaf moving direction to optimize shaping to the target volumes and/or critical organs sparing.

Using the omni-wedge in the standard head and neck irradiation technique, we achieve improved target-volumes dose homogeneity in a very significant number of clinical cases. The technique implementation is straightforward, cost-effective and has a low impact on planning and delivery times.

\section{Materials and methods}

\section{Patient selection}

We have been applying the omni-wedge technique to the irradiation of head and neck tumours since 2003. In this study we have included 156 consecutive patients with head and neck carcinoma treated in our institution. Routinely, our head and neck patients do not have targetvolumes contoured and simulation images are used for defining the treatment fields centres, dimensions and MLC shaping. During the simulation procedure (Ximatron, Varian Medical Systems), all patients were immobilized with a disposable thermoplastic head-cast. A standard field arrangement was used for all patients consisting on 2 bilateral parallel-opposed fields plus an anterior field to treat the lower neck and supraclavicular lymph nodes. The isocenter and field limits were drawn on the immobilization masks for easier patient set-up. The patients underwent a CT scan with $5 \mathrm{~mm}$ slice spacing and $3 \mathrm{~mm}$ thickness over the entire treatment area and extending at least $5 \mathrm{~cm}$ beyond target volumes in both cranial and caudal directions. Patient simulation, planning CT scan and treatment were all made in supine position. During the dosimetric planning, the standard plan and the omniwedge plan were tested and compared by visual inspection of the dose distribution on every CT image. The omniwedge plan was chosen whenever two conditions were simultaneously met: 1) the standard plan dose homogeneity was considered outside the ICRU recommendations 95-107\% [11,12] and 2) the omni-wedge plan improved the target dose homogeneity.

From these 156 patients, a representative subgroup of 15 consecutive patients was selected for a quantitative study. To allow plan inter-comparison with dose-volume histograms (DVH) the target volumes were contoured on the CT data for these 15 patients. Specifically, the lymph node levels and related CTVs were contoured on the CT slices following the DAHANCA, EORTC, GORTEC, NCIC, RTOG consensus guidelines by a radiation oncologist [13]. CTV1 was defined as the gross tumour or surgical bed with or without adjacent soft tissue (risk dependent) and involved nodal regions with or without extracapsular extension (again, risk dependent). CTV2 was defined as any elective nodal region. The PTV was then defined by adding a $3 \mathrm{~mm}$ isotropic margin to the CTVs to account for set-up uncertainties and organ motion. The spinal cord was contoured using a semi-automatic contouring tool that searches a structure outline with a $\mathrm{HU}$ threshold in every slice. We assumed a $45 \mathrm{~Gy}$ tolerance dose to the spinal cord (5\% complication probability at 5 years, $\mathrm{TD}_{5 / 5}$ ) [14].

\section{Planning techniques}

All techniques studied were based on the standard technique: bilateral parallel-opposed fields and an anterior field elected to treat the lower neck and supraclavicular lymph nodes, abutted to the lateral fields (4 or $6 \mathrm{MV}$ photons). Reaching a dose of $45 \mathrm{~Gy}$, the spinal cord is shielded in the lateral fields and the remaining dose to the spinal chain nodes is delivered using two electron fields (SSD $=100 \mathrm{~cm}$, energies of $9 \mathrm{MeV}$ or $12 \mathrm{MeV}$ ). All patients were treated to cumulative doses of 59-71 Gy.

Three set-ups were optimized and compared: (A) standard technique, (B) omni-wedge technique and (C) 3Dcustom tissue compensators technique (Fig. 1). We compared dose homogeneity on the target-volume region that includes the primary site and the upper and midcervical nodes above the shoulders (region irradiated by the lateral fields). Dose distributions were calculated with a $2.5 \mathrm{~mm}$ grid size with the Cadplan 6.4.7 (Varian Medical Systems) treatment planning system (TPS), based on the pencil beam model for $4 \mathrm{MV}$ and $6 \mathrm{MV}$ photon beams from Varian Clinac $600 \mathrm{C}$ or $2100 \mathrm{CD}$, respectively, both equipped with a multi-leaf collimator $(1 \mathrm{~cm}$ width at isocenter).

A dose homogeneity of $95-107 \%$, as recommended by the ICRU Reports [11,12] was the initial target goal for all 


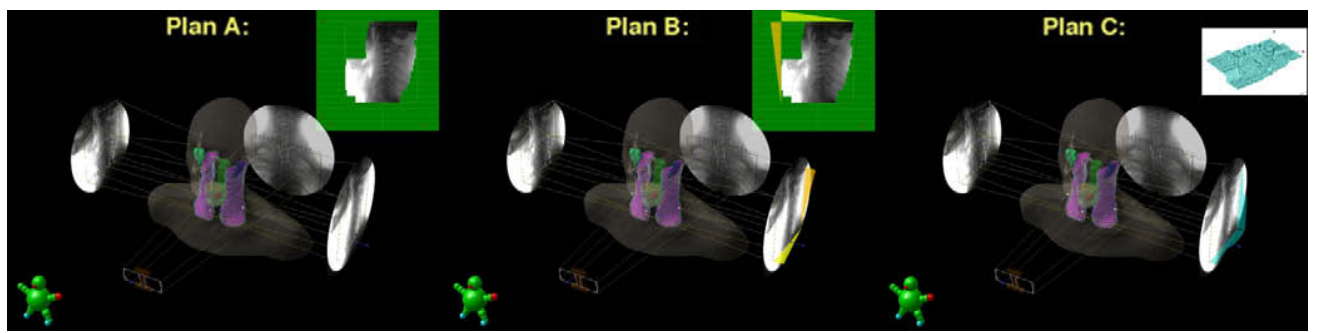

Figure 1 Schematic layout of the different techniques. (A) standard technique; (B) omni-wedge technique ; (C) 3D-tissue compensator technique.

plans. The ICRU report no 50 [11] specifies that for threedimensional calculation in large organs such as lung, liver, etc, a volume is considered clinically meaningful when its diameter exceeds $15 \mathrm{~mm}$. In our head and neck cases we defined the maximum significant dose as the dose in a sum volume equivalent to a sphere volume of $15 \mathrm{~mm}$ diameter rather than a connected region with $15 \mathrm{~mm}$ diameter, because this parameter is more easily obtained from the DVH data. This is a narrower criteria than the ICRU criteria for maximum dose in large organs. Nonetheless, the organ sizes are small in head and neck cases, justifying the use of narrower criteria. For the minimum significant dose, ICRU does not provide any recommendation and we used the same volume criteria as for the maximum significant dose.

\section{Standard technique}

The standard technique uses two isocentric bilateral parallel-opposed and coplanar photon fields. Due to head and neck shape, dose hot spots usually appear in the anterior neck region while under dosages appear in the more cranial region. Field wedging is only possible in the four orthogonal orientations since a flat junction to the anterior field is a main concern, and thus the collimator cannot be rotated and the wedge effect cannot be used in any oblique direction. For each standard plan, the lateral fields collimators were left on $0^{\circ}$ and wedges were introduced when leading to improved dose homogeneity. A third anterior field, with collimator also at $0^{\circ}$, is abutted to the lateral fields to irradiate the lower neck and supraclavicular lymph nodes.

\section{Omni-wedge technique}

As explained in the previous section, an optimal collimator rotation angle cannot be searched with the purpose of using the wedge in the best possible direction to compensate for contour irregularities and tissue non-homogeneity. The omni-wedge technique substitutes each lateral field by 2 superimposed orthogonally wedged field segments to allow the effective wedge direction to rotate, which will potentially improve target-volumes dose homogeneity. Since in most cases hot spots occur in the anterior neck region, one of the segments is wedged in order to lower the dose in this region. Furthermore, to compensate the under dosage that usually appears in the most cranial region, the second segment field is wedged to increase the dose in this region (Fig. 1). A third open field segment can be inserted when very weakly wedged fields, below the lowest nominal wedge angle available, are needed.

By varying the wedge angles and the segments relative weights, the effective wedge angle can range from zero to the maximum nominal wedge angle and the effective wedge orientation can move in the quadrant formed by the orthogonal nominal wedge orientations.

The task of choosing wedge angles and segment relative weights is performed manually through a successive approximation procedure by experienced planners. The wedge angles were chosen form the discrete values of $15^{\circ}$, $30^{\circ}, 45^{\circ}$ and $60^{\circ}$ (Varian Clinac physical wedges) and $10^{\circ}$, $15^{\circ}, 20^{\circ}, 25^{\circ}, 30^{\circ}, 45^{\circ}$ and $60^{\circ}$ (Varian Clinac enhanced dynamic wedges). For almost all patients, 2 segments in each side orthogonally wedged (4 fields total) were sufficient to improve dose homogeneity and fulfil the ICRU recommendations (Fig. 1). An algorithm guided procedure would optimize the procedure and speed it up, but it is not implemented in our department. Several algorithms were studied by other authors for this purpose [15].

As for the standard technique, an anterior field, with collimator at $0^{\circ}$, is abutted to the lateral fields to irradiate the lower neck and supraclavicular lymph nodes.

\section{D-tissue compensator technique}

The 3D-tissue compensator technique uses lateral opposed fields and a third anterior field just like the standard technique. Field compensators are added to the lateral fields to achieve optimal dose homogeneity in a given compensating plan. The TPS CadPlan uses the CT images to obtain the fluency for the compensated beam through the dose calculation of an optimal dose distribution that takes into account the effect of tissue inhomogeneities and changing anatomic contours on dose delivery. After the compensator shape calculation, the software drives an automated styrofoam milling machine which produces the depth map for the compensator according to the calculated 3D-compensator profiles. The styrofoam mould is filled with sub-millimetre iron spheres. The customized compensator, mounted on a tray, is attached to the linear accelerator gantry. By compensating tissue inhomogeneities and anatomic contours, an optimal dose homogeneity is achieved for each patient. The TPS compensator plan serves as a comparison study for the omni-wedge technique.

\section{Evaluation tools}

In the sub-group of 15 patients, a reduced PTV, PTV_r, was drawn that refers to the upper part of the target volume. Our study focuses on this part of the treatment volume which is irradiated with lateral opposed fields and our technique aims to optimize dose homogeneity in this part of 


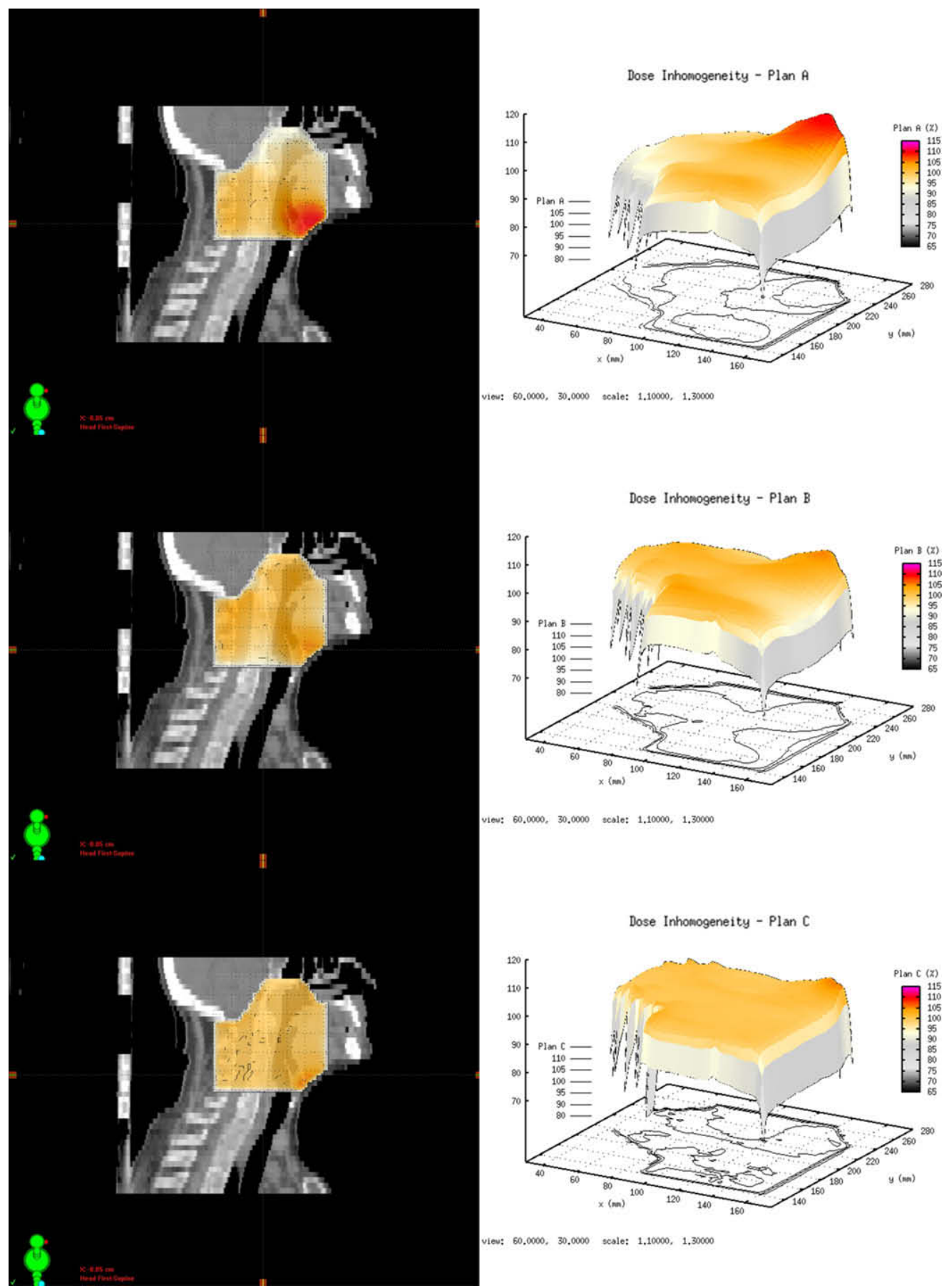

Figure 2 Example of dose distribution in the middle sagittal plane for plans A: standard technique, B: omni-wedge technique and C: using 3D-tissue compensators in a patient.

the target volumes. Dose plan normalization was initially set to the median PTV_r dose and then slightly shifted so that a good compromise between significant minimum and maximum PTV_r doses (defined in Section Planning techniques) is achieved. PTV_r minimum and maximum significant doses, volume covered by at least $95 \%$ of the dose, $V_{>95 \%}$, and volume exceeding $107 \%$ of the prescribed dose, $V_{>107 \%}$, values were extracted from the DVHs in the three techniques studied.

\section{Results}

\section{Dose distributions in example cases}

Fig. 2 shows one example of dose distribution in the middle sagittal plane for plans A: standard technique, B: omniwedge technique and C: using 3D-tissue compensators. The differences in dose homogeneity can easily be seen: the hot spots in the anterior neck region and the cold spots the 


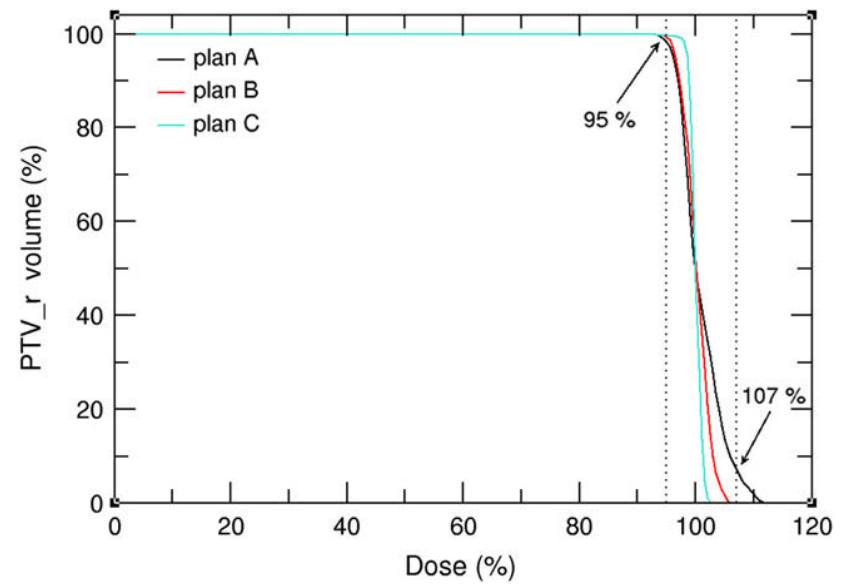

Figure 3 PTV_r DVHs in plan A, B and C for a patient.

most posterior and most cranial region fades in the omniwedge technique and in 3D-tissue compensator technique.

For this patient, Fig. 3 shows the PTV_r DVHs in plan A, B and $C$. In this case, the use of the omni-wedge technique produces a PTV_r dose homogeneity enough for fulfilling the ICRU recommendations, which were not met by the standard technique. The tissue compensator plan further enhances PTV_r dose homogeneity.

\section{PTV dose homogeneity}

Fig. 4 shows PTV_r dose range of plans A, B and C for the 15 patients selected for PTV contouring.

The number of patients for which the ICRU recommendation $95 \%$ isodose target coverage was achieved, was only $33 \%$ with the standard technique (plan A), increasing to $87 \%$ with omni-wedge technique (plan B) and to 93\% with the 3D-tissue compensator technique (plan C). Similarly, maximum significant dose exceeded the $107 \%$ ICRU recommendation in $67 \%, 20 \%$ and $13 \%$ of the studied patients when planned with plans $A, B$ and $C$, respectively.

The PTV_r volume covered by $95 \%$ of the prescribed dose, $V_{>95 \%}$, and the PTV_r volume exceeding $107 \%$ of the prescribed dose, $V_{>107 \%}$, were extracted from the DVHs to

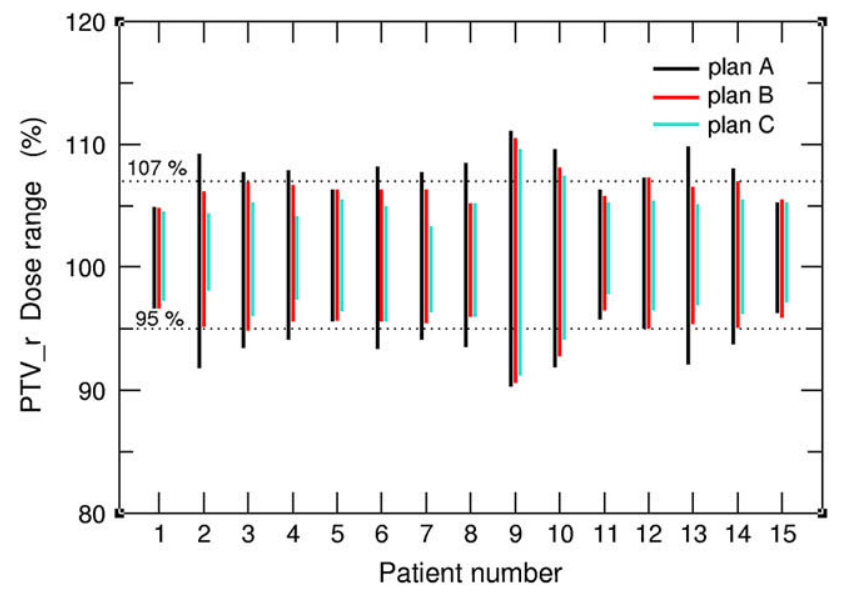

Figure 4 PTV_r Dose range in plans A, B and C, for 15 patients studied. quantify the PTV_r volume coverage and over dosage. $V_{>95 \%}$ was on average $95.4 \%$ (ranged from $85.0 \%$ to $99.5 \%$ ), $96.6 \%$ (86.0\% to $99.5 \%)$ and $97.9 \%(87.0 \%$ to $99.9 \%)$ for plans A, B and $C$, respectively. $V_{>107 \%}$ was on average $1.3 \%$ (ranged from $0 \%$ to $7.7 \%$ ) for plan $A, 0.1 \%$ (0\% to $0.2 \%)$ for plan $B$ and zero for plan $\mathrm{C}$.

These results show that there is a consistent benefit in the use of the omni-wedge technique, as the patients with PTV_r dose distribution within both ICRU dose homogeneity recommendations (95-107\%) increased from 33\% to $80 \%$. For the remaining $20 \%$ of the patients the omni-wedge technique fails with under dosage in, on average, $8.1 \%$ of the PTV_r. The over dosage was very low and considered negligible in all patients with the omni-wedge technique.

The use of the 3D-custom compensators technique would further increase this benefit as $87 \%$ of patients would become within the dose homogeneity interval goal. However, for the remaining $13 \%$ cases, the 3D compensator technique could not solve the inhomogeneity issues leading to, on average, $9.5 \%$ of PTV_r under dosage.

\section{Application of omni-wedge technique}

We have been treating patients with the omni-wedge technique since 2003. At that time, the clinical use of customized compensators was not yet implemented in our department. Until now, omni-wedge technique was chosen in $65 \%$ of 156 head and neck patients. The dependence of technique effectiveness on patient diagnostic was investigated, by sorting the patients into groups according to their tumour location.

Fig. 5 shows the distribution of the number of patients treated with standard technique and with omni-wedge technique, per diagnostic group. The oral cavity group shows the highest percentage (95\%), of patients for whom the omni-wedge technique was chosen. In the other 4 groups, this technique was selected in roughly $50 \%$ or more of the cases. From head and neck tumour sites, the oral cavity has the more anterior location, which enhances the occurrences of hot spots in that region, justifying the need for a more complex dose shape to compensate for the more pronounced contour irregularities.

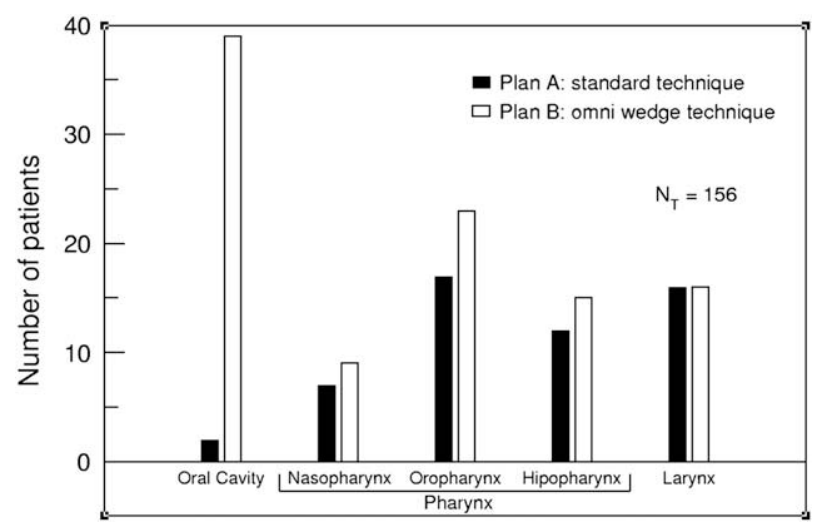

Figure 5 Number of patients treated with the standard technique (Plan A) and omni-wedge technique (Plan B), grouped into diagnostic groups. 
Within the five different clinical groups, the oral cavity group benefits the most of this technique. Furthermore these results show that, in all diagnostic groups, the omniwedge technique should be tested and compared to the standard as it provides improved dose homogeneity in at least $50 \%$ of the cases.

\section{Discussion}

The analysis presented in the previous sections aimed at assessing the potential benefits of the omni-wedge technique in dose homogeneity in the treatment of head and neck tumours, when no 3D-custom compensator system or IMRT are available in a radiotherapy department. We have compared treatment planning among three planning techniques. The results show that the omni-wedge technique improves PTV dose homogeneity towards values which were sufficient in $80 \%$ of the studied cases to fulfil the ICRU homogeneity recommendations, 95-107\%. This study only focus in the upper part of treatment volumes irradiated with lateral opposed fields. We have presented both qualitative and quantitative evidence supporting the application of this technique in head and neck tumours during the first treatment phase.

This technique is simple enough to apply in the routine work flow of a radiotherapy department. It has the disadvantage of increasing the number of treatment fields (although using the same field gantry incidence) and consequently increasing the delivery times. In most cases, two field segments orthogonally wedged were considered enough. When very weakly wedged fields, below the lowest nominal wedge angle available, are needed, a third open field segment can be added. We have estimated that the increase of the delivery time is only approximately $20 \%$.

The routine implementation of this technique is straightforward and easily achieved with normal radiotherapy equipment and treatment planning systems. Also, this technique is cost-effective and circumvents the unavailability of customized tissue compensators in most institutions. In this paper, we detail our experience with the omni-wedge technique in head and neck tumour irradiation in the last 5 years. We currently use this technique also in conformal head and neck irradiation techniques, and in other tumour locations. Other location where it is used in our department with considerable advantages is the whole breast irradiation. Specifically, the radiotherapy for the breast with regional node involvement has also the same limitation on collimator rotation. Tangential breast beams have a $0^{\circ}$ collimator rotation to provide a flat junction to the supraclavicular fields. The breast contour anatomic irregularities often benefit from the composed wedge dose effect in both orthogonal directions, which can be achieved with the omni-wedge technique.

\section{Acknowledgements}

The authors wish to express their gratitude to Ana Cristina Cleto, Radiotherapy Oncologist at the Radiotherapy
Department, Hospitais da Universidade de Coimbra, for her work on delineating target volumes and valuable discussions.

\section{References}

[1] Cozzi L, Fogliata A, Lomax A, Bolsi A. A treatment planning comparison of 3D conformal therapy, intensity modulated photon therapy and proton therapy for treatment of advanced head and neck tumours. Radiother Oncol 2001; 61(3):287-97.

[2] Cozzi L, Fogliata A, Bolsi A, Nicolini G, Bernier J. Threedimensional conformal vs. intensity-modulated radiotherapy in head-and-neck cancer patients: comparative analysis of dosimetric and technical parameters. Int J Radiat Oncol Biol Phys 2004;58(2):617-24.

[3] Sultanem K, Shu HK, Xia P, Akazawa C, Quivey JM, Verhey LJ, et al. Three-dimensional intensity-modulated radiotherapy in the treatment of nasopharyngeal carcinoma: the University of California-San Francisco experience. Int J Radiat Oncol Biol Phys 2000;48(3):711-22.

[4] Harari PM, Sharda NN, Brock LK, Paliwal BR. Improving dose homogeneity in routine head and neck radiotherapy with custom 3-D compensation. Radiother Oncol 1998;49(1): 67-71.

[5] Sharma SC, Johnson MW. Clinical considerations in the use of missing tissue compensators for head and neck cases. Med Dosim 1998;23(4):267-70.

[6] Chang SX, Cullip TJ, Deschesne KM, Miller EP, Rosenman JG. Compensators: an alternative IMRT delivery technique. J Appl Clin Med Phys 2004;5(3):15-36.

[7] Dobbs J, Barret A, Ash D. Practical radiotherapy planning. 3rd ed. Arnold Publishers; 1999.

[8] Sohn JW, Suh JH, Pohar S. A method for delivering accurate and uniform radiation dosages to the head and neck with asymmetric collimators and a single isocenter. Int $\mathrm{J}$ Radiat Oncol Biol Phys 1995;32(3):809-13.

[9] Milliken BD, Hamilton RJ, Rubin SJ. The omni wedge: a method to produce wedged fields at arbitrary orientations. Med Phys 1996;23(3):337-42.

[10] Milliken BD, Turian JV, Hamilton RJ, Rubin SJ, Kuchnir FT, Yu CX, et al. Verification of the omni wedge technique. Med Phys 1998;25(8):1419-23.

[11] ICRU prescribing, recording and reporting photon beam therapy report 50. Washington, DC: International Commission on Radiation Units and Measurements; 1993.

[12] ICRU prescribing, recording and reporting photon beam therapy (supplement to ICRU report 50) report 62. Washington, DC: International Commission on Radiation Units and Measurements; 1999.

[13] Grégoire V, Levendag $P$, Ang KK, Bernier J, Braaksma $M$, Budach V, et al. CT-based delineation of lymph node levels and related CTVs in the node-negative neck: DAHANCA, EORTC, GORTEC, NCIC, RTOG consensus guidelines. Radiother Oncol 2003;69:227-36.

[14] Emami B, Lyman J, Brown A, Coia L, Goitein M, Munzenrider JE, et al. Tolerance of normal tissue to therapeutic irradiation. Int J Radiat Oncol Biol Phys 1991;21(1): $109-22$.

[15] Dai J, Zhu Y. Comparison of two algorithms for determining beam weights and wedge filters. J Appl Clin Med Phys 2002; 3(3):190-9. 